Revue des patrimoines

\title{
La montagne, lieu de développement industriel : l'exemple du Languedoc-Roussillon
}

\section{(2) OpenEdition \\ Journals}

Édition électronique

URL : http://journals.openedition.org/insitu/3412

DOI : 10.4000/insitu.3412

ISSN : 1630-7305

Éditeur

Ministère de la culture

\section{Référence électronique}

"La montagne, lieu de développement industriel : l'exemple du Languedoc-Roussillon », In Situ [En ligne], 8 | 2007, mis en ligne le 01 mars 2007, consulté le 19 avril 2019. URL : http:// journals.openedition.org/insitu/3412; DOI : 10.4000/insitu.3412

Ce document a été généré automatiquement le 19 avril 2019

\section{(c) (1) $(9$}

In Situ Revues des patrimoines est mis à disposition selon les termes de la licence Creative Commons Attribution - Pas d'Utilisation Commerciale - Pas de Modification 4.0 International. 


\section{La montagne, lieu de développement industriel : l'exemple du Languedoc- Roussillon}

1 Dans la société du début du XXIe siècle, l'espace montagnard apparaît le plus souvent comme une ruralité protégée, caractérisé par une grande qualité de vie offrant calme et air pur, avec une densité de population faible et dont ces dernières décennies ont consacré la «vocation » de loisirs dans un environnement qualifié de naturel. Il y a 150 ans, on y voyait plutôt des régions reculées - difficiles, hostiles même - surpeuplées de paysans pauvres: les sommets sont "affreux ", les crêtes déchiquetées, les versants rocheux... C'est un espace sous-développé, donc potentiellement pionnier que dynamisme et capitaux peuvent contribuer à mettre en valeur. Quelle que soit leur nature, les projets de développement (mines, barrages, usines, reboisement...) insistent toujours sur le mieux-être qu'ils vont apporter à une population nombreuse qui a du mal à survivre en dépit d'une ardeur au travail largement reconnue.

2 Par contre, malgré une certaine marginalité, la montagne européenne n'est pas une terra incognita à la manière de l'Ouest américain : jusqu'à plus de 2500 mètres d'altitude, elle est parcourue, habitée, mise en valeur par des hommes, bûcherons et éleveurs en particulier.

3 La grande mutation industrielle du XIXe siècle n'ignore pas la montagne qui évolue parfois même plus vite que la campagne de plaine. La transformation y revêt toutefois des caractères particuliers que nous allons analyser, le plus important étant l'opposition entre vallées et versants.

4 Bordé de montagnes moyennes, le Languedoc-Roussillon permet de comparer deux dynamiques franchement divergentes au cours du XIXe siècle : des plaines et des bassins de basse altitude qui basculent progressivement d'une céréaliculture dominante vers la quasi-monoculture de la vigne tandis que les vallées de montagne se tournent résolument vers l'industrie textile et métallurgique et que les hauts versants sont les premiers à connaître déclin et exode rural. Comme presque partout, il y a eu beaucoup plus d'usines dans les vallées de montagne que dans les plaines plus spontanément tournées vers l'agriculture. 


\section{La montagne, productrice d'énergie}

Les innombrables rivières et ruisseaux de montagne constituent une source inépuisable d'énergie mécanique or la « révolution industrielle » est d'abord celle de la maîtrise de l'énergie, surtout dans un pays comme le Sud de la France, relativement pauvre en charbon (fig. $\mathbf{n}^{\circ} \mathbf{1}$ ). Bien qu'apparue seulement au début du XXe siècle, l'expression « houille blanche » montre bien cette équivalence pratique entre chute d'eau et charbon.

Figure 1

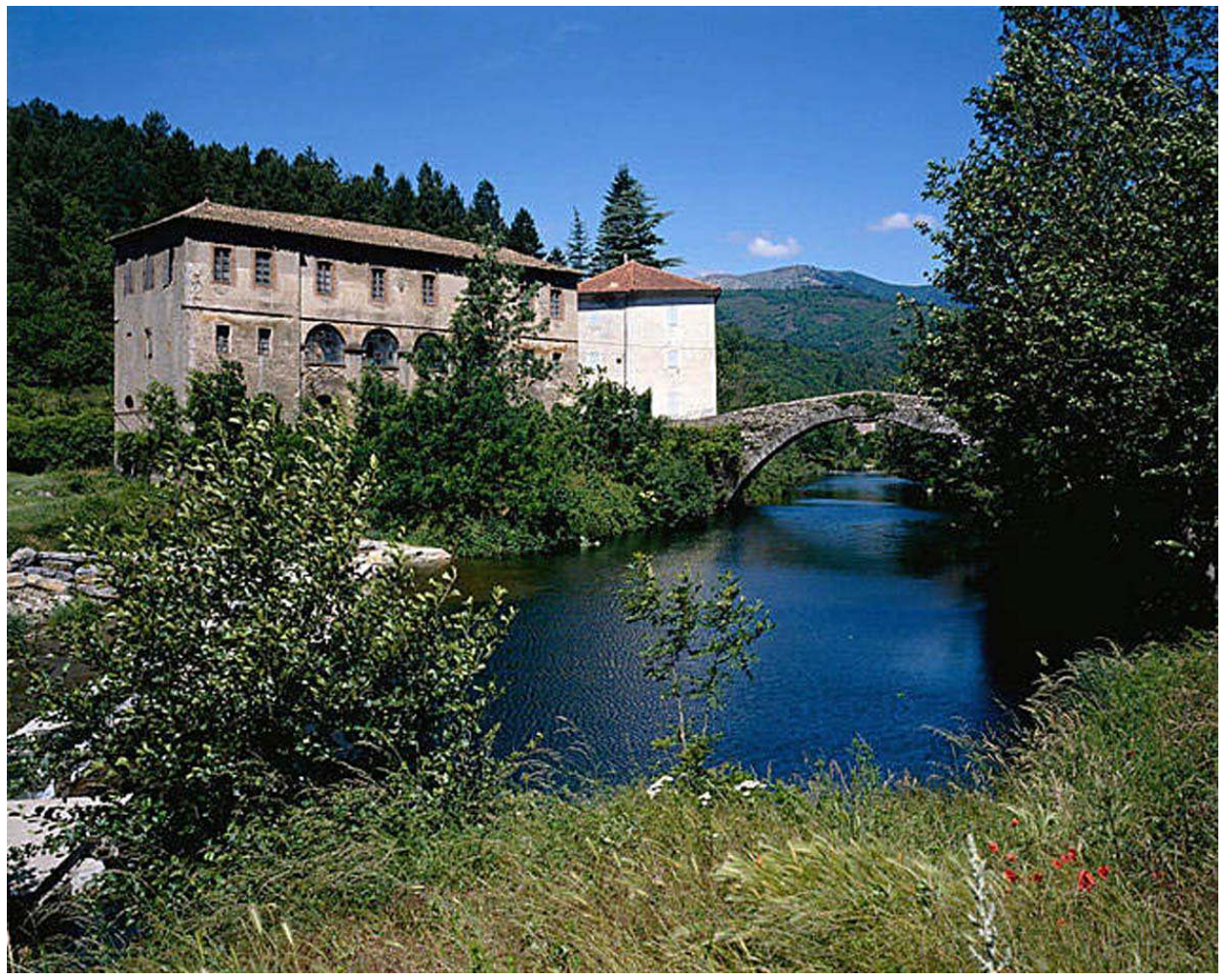

Usine et rivière : filature de soie du Pont de Rastel à Chamborigaud (Gard). Périn, Jean-Michel (C) Inventaire général, ADAGP, 1990.

6 Jusqu'au début du XIXe siècle, ces cours d'eau n'animaient que de petites «usines" directement liées aux productions et souvent à la consommation locales : moulins divers (à farine, gruau, orge mondé, huile...), foulons liés au tissage artisanal local, etc. Seules les usines métallurgiques, en particulier celles à fer comme les célèbres forges dites «catalanes » et les martinets qui les accompagnent, produisent pour un marché allant bien au-delà de la vallée. La grande production textile est celle d'une main-d'œuvre urbaine ou périurbaine: Carcassonne pour le drap de laine, Nîmes pour le coton, Le Vigan, Alès ou Aubenas pour la soie.

7 Dans les décennies 1820-1850, la saturation des sites hydrauliques de plaine provoque des installations qui remontent de plus en plus vers l'amont, souvent en partant des villages existants. Le textile domine largement avec l'arrivée des machines à carder et à filer, suivies un peu plus tard du tissage et du tricotage, longtemps monopole d'un artisanat urbain ou villageois spécialisé. La bergère et son fuseau cèdent progressivement la place à 
l'ouvrière. Des Ardennes au massif des Arbailles ${ }^{1}$, les vallées de modestes ruisseaux se transforment en chapelets d'usines, en commençant par les plus faciles d'accès. Roues à aubes et à augets se multiplient d'année en année (fig. $\mathbf{n}^{\circ} 2$ ).

Figure 2

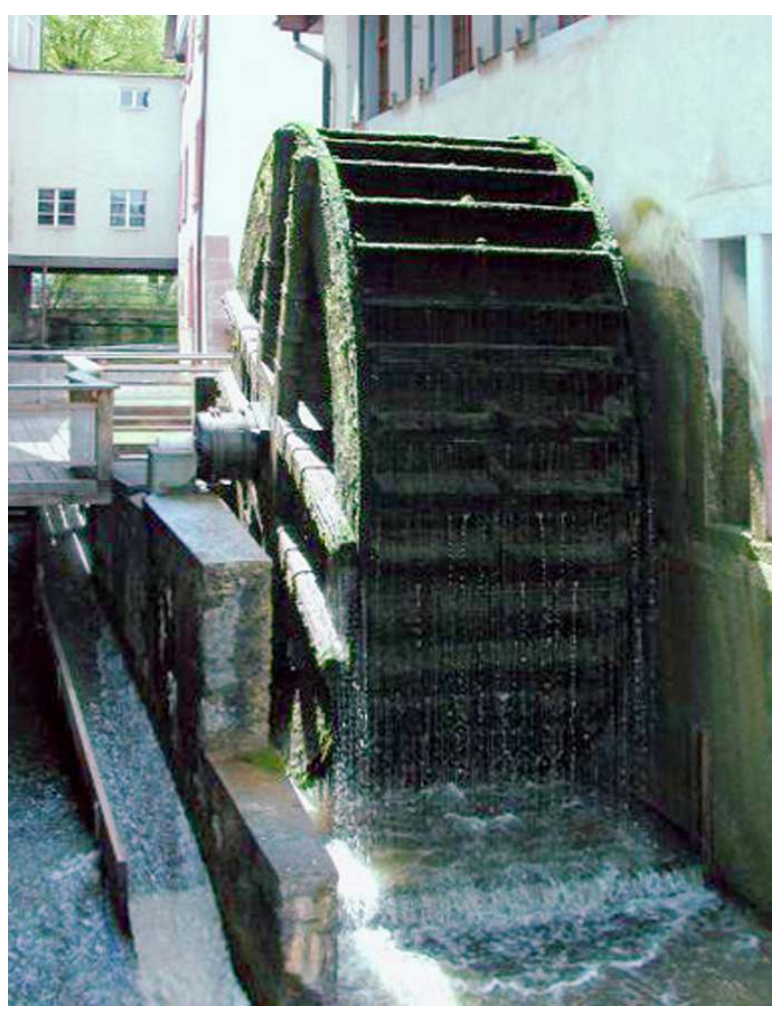

Roue à aube dans une mégisserie de la Montagne Noire audoise. Wienin, Michel (c) Wienin, Michel, 1996.

Bien plus encore que celui du fil, produit léger et facile à fractionner, le déplacement des machines nécessite des chemins carrossables et de gros travaux pour leur réalisation. Véritable chantier national, le désenclavement des villages reculés favorise les échanges et donne du travail à une population importante. Il y a une complémentarité évidente : l'industrie a besoin de voies de communication, routes et voies ferrées favorisent à leur tour le développement des entreprises. Un bel exemple de ce désenclavement est donné par les moulins dont les meules, jadis en grès ou granite de pays, sont remplacées au fur et à mesure de l'avancée du « progrès » par des produits plus performants, le plus souvent en meulière de La Ferté-sous-Jouarre (Seine-et-Marne), souvent formées de secteurs pour en faciliter le transport et assemblées par un cerclage de fer. En même temps, des scieries ou des papeteries s'installent dans presque toutes les vallées (fig. $\mathbf{n}^{\circ} \mathbf{3}$ ). 
Figure 3

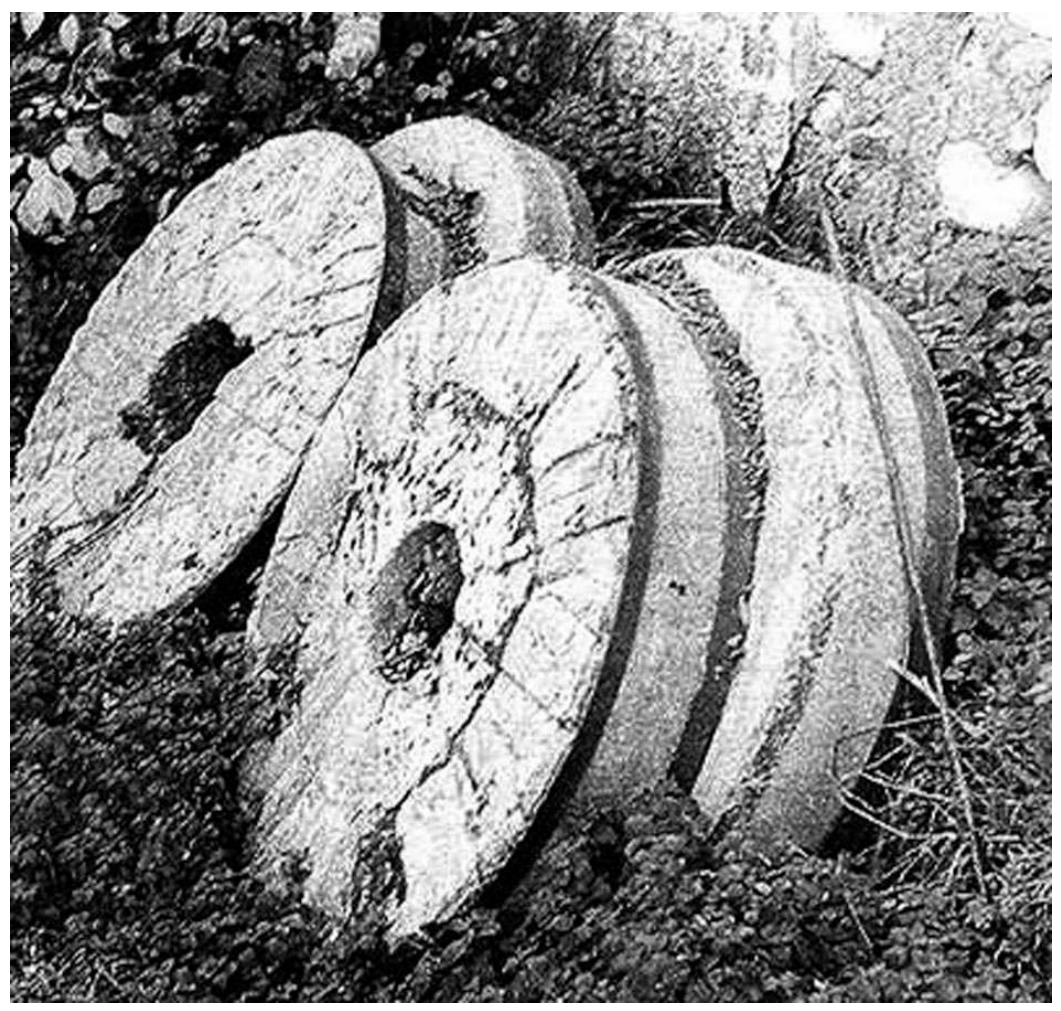

Meules assemblées et cerclées. Wienin, Michel

(c) Wienin, Michel, 1991.

9 A partir de la fin du XIXe siècle, le développement rapide de l'hydro-électricité bouleverse la situation. Jusque vers 1900 , on produit du courant continu qui ne peut se transporter au-delà de quelques kilomètres sans d'importantes pertes de charge. Ensuite, c'est la faiblesse d'un réseau de distribution encore embryonnaire qui limite la distribution et les premières centrales sont généralement attachées à une usine consommatrice. Cela concerne d'ailleurs souvent des technologies à la pointe du progrès : chimie, électrométallurgie comme l'aluminium dans les Alpes ou les aciers spéciaux à Saint-Chély-d'Apcher (Lozère). Les villages tendent à se transformer en petites villes ouvrières et sont souvent éclairés à l'électricité quand Paris l'est encore au gaz, phase qu'ils n'ont jamais connue (fig. $n^{\circ} 4$ ). 


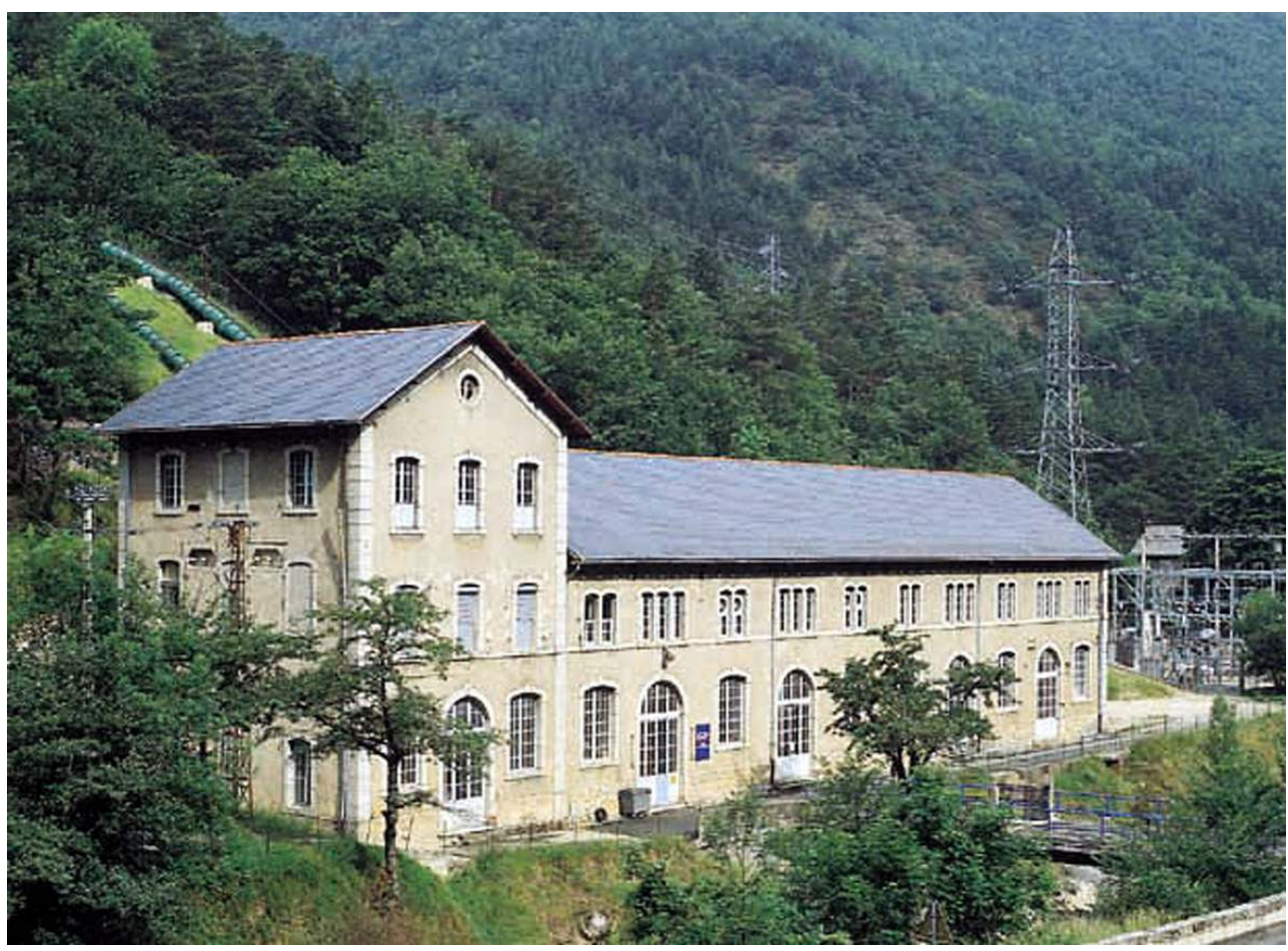

Centrale hydroélectrique de Saint-Georges à Axat (Aude) : mise en service en 1900. Périn, Jean-Michel (c) Inventaire général, ADAGP, 1997

\section{Les productions traditionnelles}

Outre quelques productions agricoles alimentaires un peu marginales dans la consommation urbaine (charcuterie, fromages, châtaignes...), la montagne approvisionne depuis longtemps les villes dans deux grands groupes de produits : le bois et les produits d'élevage.

\section{Le bois et ses dérivés}

11 La situation au début du XIXe siècle est celle d'une activité de cueillette entravée par les difficultés excessives du transport.

12 Le bois d'œuvre (charpenterie et menuiserie) est le plus souvent transporté par flottage quand les cours d'eau le permettent. C'est une exploitation de matière première sans grand profit pour les villages. Le bois de feu n'est guère qu'un sous-produit à usage local. Pour l'exportation vers les villes, il est généralement transformé d'abord en un produit plus léger: le charbon (de bois), aussi indispensable pour l'usage domestique qu'industriel.

13 Dans la mentalité du montagnard de cette époque, la forêt est un espace non exploité, refuge, qui plus est, d'animaux nuisibles (loups, ours...). La défricher, empêcher les arbres de repousser, convertir cet espace inutile en prairies qui permettront d'augmenter le cheptel, c'est œuvrer pour l'économie et même la civilisation. On sait à quelle désertification des massifs cette vision des choses a conduit et combien le reboisement 
qui ne pouvait être rentable que globalement et à long terme a été difficile à faire accepter par les populations locales.

Généralement effectués loin des villages par des équipes d'hommes spécialisés, mais marginaux par rapport à la population, bûcheronnage et charbonnage apportent peu aux communautés et n'évoluent guère avant l'arrivée des premières voies ferrées dans la seconde moitié du XIXe siècle. Favorisé par une facilité d'exportation nouvelle, le premier se développe là où subsistent des forêts exploitables tandis que le second décline devant la concurrence de la houille.

Le train bouleverse la filière bois : le nouveau mode de transport ne se contente pas de diminuer sensiblement le prix du bois vendu en ville, il en améliore la qualité par disparition du «maquage " (les chocs contre les rochers dans les zones de rapides) et permet surtout le transport en planches; la chaîne économique est entièrement modifiée : le sciage manuel, près des ports fluviaux, est abandonné au profit du sciage mécanique, effectué dans des scieries hydrauliques qui se multiplient alors dans toutes les vallées. Le volume de matériau acheminé augmente rapidement, répondant à une demande nouvelle dans l'ameublement comme dans l'architecture: les inventaires montrent la multiplication des meubles, leur taille plus grande et l'observation des constructions la multiplication des fermes de charpente, des huisseries, des cloisons en bois, etc. Dans une région comme le Languedoc où ce matériau était rare, le changement est sensible. Entre 1880 et 1900 , les scieries sont devenues la principale activité économique non agricole de nombreux villages. A Lapradelle-Puylaurens, dans les Pyrénées audoises, elles sont trois, alignées le long de la voie ferrée de Carcassonne à Perpignan et emploient le tiers de la population active !

16 Jadis destinés au charbonnage et au fagotage, les bois moyens et petits trouvent un nouveau débouché dans la papeterie où les traditionnels chiffons cèdent la place, à partir de 1870, au bois blanc déchiqueté. Dans les régions où les feuillus sont plus abondants que les résineux, d'Anduze (Gard) à Axat (Aude) en passant par la Montagne Noire, le cartonnage l'emporte sur le papier fin, surtout quand des industries consommatrices d'emballages existent au voisinage, la chapellerie ou la mégisserie en particulier. 
Figure 5

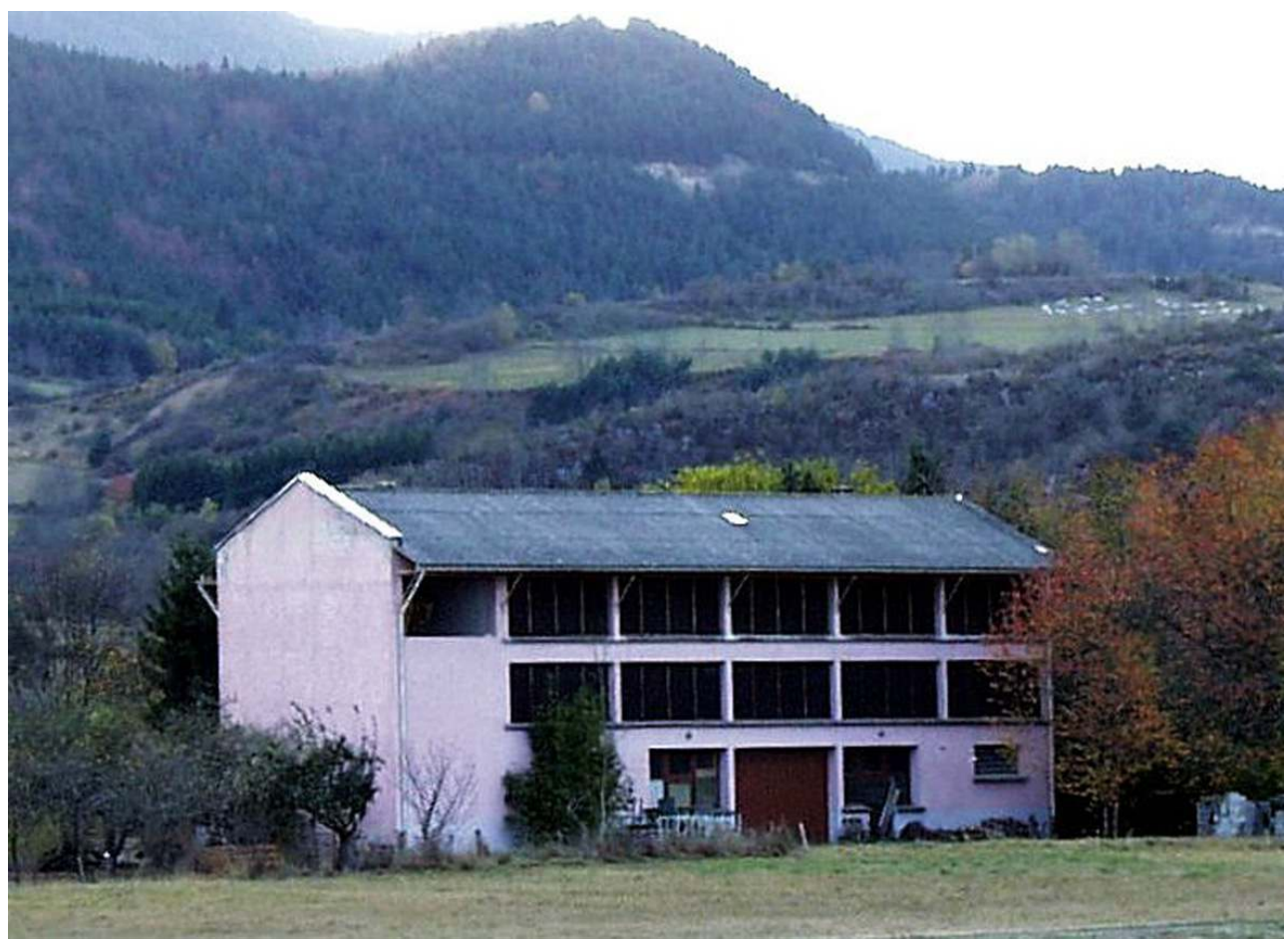

Sécherie de graines d'Espezel, dans les Pyrénées audoises. Wienin, Michel (c) Wienin, Michel, 2001.

17 Plus rarement, on trouve des sécheries industrielles de graines destinées au reboisement (fig. $n^{\circ} 5$ ).

\section{La laine}

18 Elle est le co-produit par excellence de l'élevage. De plus en plus souvent transformée sur place, elle joue un rôle majeur dans l'industrialisation des vallées de montagne et constitue quasiment partout l'industrie caractéristique de la montagne. 
Figure 6
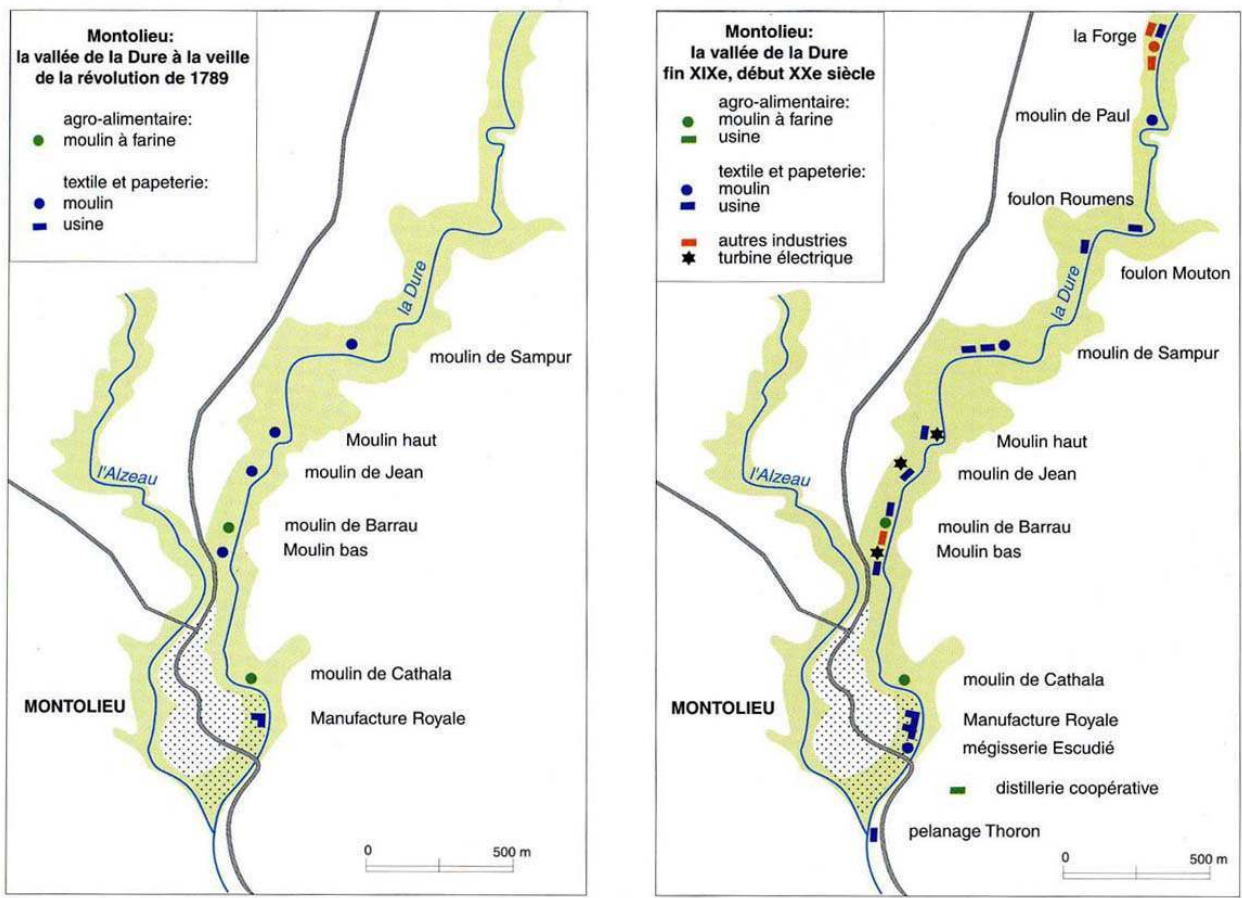

La colonisation industrielle de Montolieu et de la vallée de la Dure (Aude) dans la Montagne Noire, vers 1800 et vers 1900. Wienin M., 1998, Le patrimoine industriel de l'Aude, Images du Patrimoine, $n^{\circ} 177$, p. 5.

19 Jusqu'à l'Empire, le filage est le point noir de l'ensemble du processus textile. Il constitue l'activité féminine par excellence mais l'arrivée des premières machines à carder et à filer à partir de 1810 change tout; la machine et l'énergie remplacent progressivement la main-d'œuvre : les filatures industrielles, parfois simplement appelées les « mécaniques » colonisent les vallées dans une dynamique en trois phases (fig. $\mathbf{n}^{\circ} \mathbf{6}$ ).

Des négociants urbains créent des établissements assez importants, souvent à partir d'un gros moulin ou d'une papeterie plus anciens ; des propriétaires de moulins, souvent des moulins à foulon, complètent leur établissement par un atelier de filature dont la taille dépend de la puissance énergétique disponible et de leurs moyens financiers, de nouvelles usines enfin sont créées ex nihilo de plus en plus loin des villages. 
Figure 7

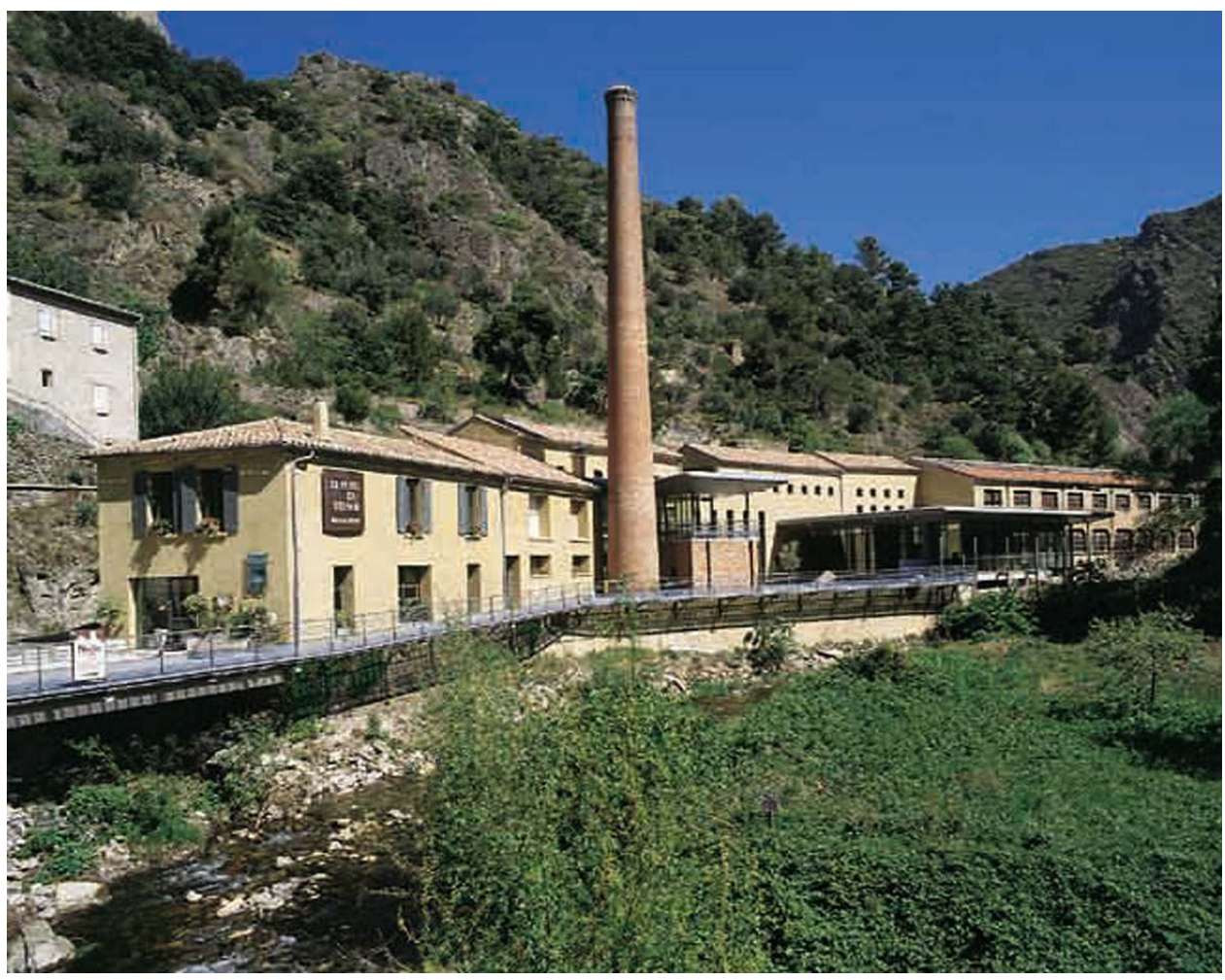

Usine lainière comprenant toutes les phases à Lastours (Aude). Périn, Jean-Michel

(c) Inventaire général, ADAGP, 1997.

Dans un second temps, ce sont les autres opérations textiles qui se multiplient dans les vallées de montagne : tissage, tricotage et surtout foulonnage, teinture, apprêts ou encore fabrication de feutre, en particulier pour la chapellerie à Espéraza (Aude), mais aussi à Anduze (Gard), ou à Meyrueis (Lozère) (fig. $\mathbf{n}^{\circ}$ 7).

On peut identifier plusieurs types d'implantations :

- les ateliers isolés, implantés en chapelet le long de ruisseaux à débit modeste mais à pente assez forte, se succèdent en rentabilisant au mieux le potentiel hydraulique : le canal de fuite de chaque usine alimente en général directement la prise d'eau de l'usine située immédiatement en aval ; on peut parler d'aménagement en escalier, sur plusieurs dizaines de kilomètres, parfois. Les vallées qui descendent des sommets à climat subatlantique de la Montagne Noire, au nord-ouest de Carcassonne, sont particulièrement caractéristiques de cette disposition ;

- les regroupements urbains, parfois le long d'un grand béal (canal de dérivation) d'origine ancienne sont relativement rares et limités à des rivières à débit suffisant mais de pente plus faible : Marvejols (Lozère), Lodève (Hérault), la chapellerie d'Espéraza déjà citée, etc. ;

- les gros établissements isolés sont exceptionnels. Localement, la présence d'équipements annexes (logements d'ouvriers, église, école, magasins...) tend vers le modèle de la colonie industrielle (usines de soie de Pont-d'Hérault, à Sumène, Gard).

La sociologie de cette industrie textile de montagne est un peu particulière: la maind'œuvre est très largement féminine, en particulier pour la filature. Si les hommes sont en général de « vrais » ouvriers dont c'est l'activité principale pendant l'ensemble de la 
vie active, les femmes, nettement moins payées malgré des conditions et un travail aussi pénibles, n'y trouvent souvent qu'un complément au revenu agricole ou salarié de leur famille: jeunes filles qui «se font leur trousseau», jeunes femmes sans enfants dont l'apport sera souvent investi en terre ou en murs, veuves rembauchées "par charité ", etc.

Paradoxalement, cette industrialisation qui apporte une masse d'argent non négligeable dans les villages, favorisant de manière importante ceux où elle s'implante, accélère l'exode rural des autres par l'appel de population ouvrière qu'elle constitue mais surtout par l'effondrement du prix du fil qui l'accompagne: en quelques années, filature manuelle et tissage artisanal perdent toute rentabilité, privant les paysans les plus pauvres d'un appoint d'argent essentiel. L'eau, indispensable dans le processus de fabrication (lavage, teinturerie, foulonnage...) et incontournable source d'énergie, impose que, dans les vallées, l'industrialisation textile s'étire le long des rivières, ignorant pratiquement villages et hameaux de versants, pourtant bien mieux exposés, et, a fortiori, ceux de crête ou de plateau. Elle favorise la concentration de la population dans les fonds de vallées aux dépens des hautes terres. Ce phénomène est parfaitement lisible dans le paysage de tous les massifs dont le dénivelé dépasse 200 à 300 mètres seulement.

\section{Les autres productions de l'agriculture traditionnelle}

Ces dernières ne sont pas toujours en reste. L'amélioration des conditions de conservation et surtout de transport, en particulier le développement d'une véritable chaîne du froid, se traduisent par une spécialisation de la montagne vers une agriculture qui s'intègre dans une économie d'échanges nationale puis internationale au fur et à mesure que les villes se développent et que les voies ferrées pénètrent dans les vallées. L'agriculture, essentiellement de subsistance (céréales pauvres comme le seigle, sarrasin ou cultures vivrières, pommes de terre), s'efface progressivement au bénéfice de productions destinées aux villes comme les produits laitiers, avec tout d'abord les fromages, recommandés par les médecins et les hygiénistes dès le XVIIIe siècle. Ce sont ceux, pressés, à pâte cuite, pouvant se conserver longtemps, qui se développent en premier.

Le beurre, dont l'utilisation constituait dans le Midi un signe extérieur d'appartenance aux classes aisées, voit sa consommation croître avec le développement de la bourgeoisie urbaine. Bien loin de son aire traditionnelle, il devient, avant 1900, une grande spécialité de la Cerdagne, haut plateau pyrénéen, à destination de Perpignan et au-delà, de Narbonne, Montpellier et même Barcelone. Il convient toutefois de noter qu'avant la Première Guerre mondiale, la production demeure entièrement domestique ou artisanale. Le lait, dont la conservation et le transport sont difficiles, est consommé quasiexclusivement par les enfants jusque vers 1950 et distribué en vrac; il est traditionnellement produit le plus près possible de son lieu de consommation, donc autour des villes. Ce sont les progrès dans sa conservation et son conditionnement qui bouleversent la structure de distribution : à partir de 1960, coopératives ou sociétés de ramassage ont seules les moyens d'investir en équipements de plus en plus coûteux et de les rentabiliser. C'est l'âge d'or de l'élevage laitier de montagne. Par contre, le mouvement de concentration qui lui fait suite entraîne des regroupements et l'abandon de nombreux petits centres. traditionnellement «sur pieds", c'est-à-dire à pied, vers les boucheries urbaines, 
remplacées au cours du XIXe siècle par des abattoirs souvent municipaux. Là aussi, la multiplication des transports et la maitrise du froid favorisent l'implantation de l'abattage près de la production et une distribution en quartiers débités et parfois en pièces préparées (rôtis). Un bel exemple d'abattoir de ce type, indépendant de la zone de consommation, se trouve à Langogne, au nord de la Lozère, au centre d'une zone d'élevage, mais surtout à côté de la voie ferrée de Nîmes à Clermont-Ferrand et, au-delà, vers Montpellier, Marseille et Paris.

Co-produit incontournable de l'abattage, le cuir «suit le mouvement». Tanneries et mégisseries, jadis urbaines et repoussées au cours du XIXe siècle de plus en plus loin des centres-villes, cèdent petit à petit la place à des usines isolées et donc moins gênantes pour la population, disposant d'eau plus abondante et plus pure. A Langogne, trois mégisseries sont établies autour de 1930 en lien évident avec les abattoirs cités plus haut.

\section{Une production agro-industrielle : la soie}

Dans de nombreuses régions, des spécialisations agricoles font leur apparition à partir du XVIIe siècle. Il s'agit souvent de cultures destinées à l'industrie textile : chanvre, lin, pastel. Le phénomène est rare en montagne avec toutefois l'exemple assez extraordinaire de la soie dans les Cévennes. Pratiquées depuis plusieurs siècles de l'Hérault à l'Ardèche, la culture du mûrier et l'«éducation" (élevage) des vers à soie connaissent un développement fulgurant dans la première moitié du XIXe siècle. Exemple quasi unique d'une région montagneuse basculant précocement vers une agriculture industrielle, l'épopée de la soie cévenole a fait couler beaucoup d'encre - on pensera par exemple à la thèse de Max Weber sur le rôle du protestantisme, très implanté localement, dans ce qu'il interprète comme un proto-capitalisme - mais cette industrie bénéficie entre autres de deux facteurs externes : le développement de la demande lié à la « démocratisation » du luxe grâce à l'essor d'une bourgeoisie d'affaires d'une part, la mise au point du chauffage à la vapeur des bassines de filature en 1807, puis l'emploi des machines à vapeur pour entrainer les dévidoirs, d'autre part. En quelques décennies, des millions de mûriers envahissent les versants jusqu'à près de $900 \mathrm{~m}$ d'altitude entre Le Vigan (Gard) et Aubenas (Ardèche), les fermes s'accroissent de magnaneries parfois plus grandes qu'elles et toute la population féminine disponible est embauchée comme main-d'œuvre quelques semaines à quelques mois par an.

Comme celles de laine, les filatures de soie sont installées en bord de rivières, participant largement au développement des villages de vallées. Par contre, étroitement associée à une pratique agricole occupant de vastes superficies, c'est une production qui favorise la densification de l'espace rural proprement dit conduisant à cette situation paradoxale que les villages du nord du Gard ou du sud de la Lozère avaient en 1850 une densité de population supérieure à celle de la Beauce !

\section{Les industries liées à la mine}

Avant que les progrès techniques, en particulier dans le pompage des eaux d'infiltration, n'aient permis l'exploitation des richesses du sous-sol des plaines, la mine apparait normalement liée à la montagne qui recèle les gisements métalliques à tel point que divers mots allemands pour mine (Bergwerk) et mineur (Bergmann, Bergarbeiter) signifient littéralement «usine, homme, travailleur de la montagne». Longtemps, l'image du 
mineur reste associée à celle des rochers près desquels s'ouvre sa galerie et le traitement des produits est presque exclusivement effectué sur place. L'Ariège produit du fer et des hommes, disait Napoléon mais s'il est assez facile de déplacer les seconds, le minerai ne peut être extrait que là où la nature l'a rendu accessible. Dans le domaine minier, la "révolution industrielle» se traduit par un bouleversement du mode d'extraction: le principe de trier sur place (scheidage) et de ne ressortir que ce qui est utilisable cède la place à une technique rendue possible par la maîtrise de l'énergie : tout est ressorti en vrac et on traite le minerai dehors (concassage, triage, lavage), d'abord manuellement puis de manière mécanique. Ces opérations nécessitent toujours une quantité importante d'eau et les ateliers de traitement quittent progressivement le carreau de la mine pour s'installer en fond de vallée.

\section{Les grandes concentrations minérales}

Il s'agit principalement de la houille et du minerai de fer dont les gisements vraiment importants (plusieurs centaines de millions de tonnes) se situent sous les plaines et les bas plateaux du Nord et du Nord-est de l'hexagone. La première est également présente dans plusieurs dizaines de bassins à la périphérie du Massif Central et des autres massifs hercyniens, dans la partie basse des vallées plutôt que sur les versants de montagnes importantes. Dans les secteurs où les couches carbonifères affleurent, elle constitue des volumes conséquents qui se mesurent en millions de tonnes et leur exploitation bouleverse le paysage et la société.

Le fer abonde particulièrement dans l'Est de la France mais aussi dans de nombreuses régions de collines et de piémont. Les gisements se trouvent assez souvent au voisinage de ceux de houille car le traitement du fer nécessite l'usage de coke, sous-produit du charbon. La complémentarité houille et minerai de fer est à l'origine des usines sidérurgiques et métallurgiques qui forment un ensemble cohérent à la base de toute l'industrie lourde du XIXe siècle (fig. $\mathbf{n}^{\circ} \mathbf{8}$ ). 


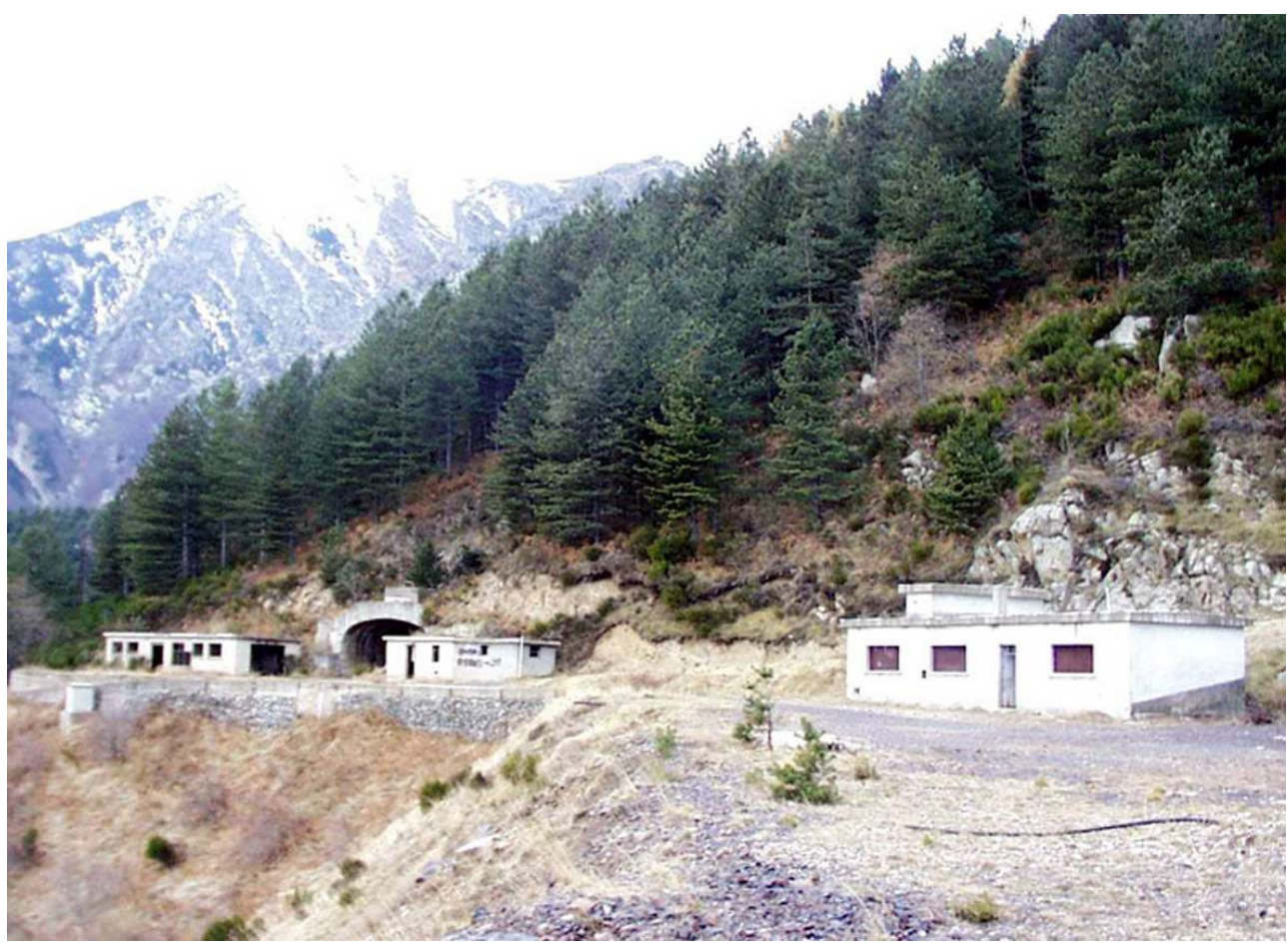

Carreau de la mine de fer de Batère, dans le massif du Canigou, Corsavy, (Pyrénées Orientales.). Wienin, Michel

(c) Wienin, Michel, 2002.

Le développement massif de ces grands sites « mino-métallurgiques» est assez tardif (à partir de 1820) et les investissements importants qu'ils nécessitent alors sont à l'origine d'ensembles industriels dont la dimension nous étonne encore, véritables colonies industrielles sans lien direct avec l'environnement montagnard.

Sociologiquement, la campagne relativement proche est incapable de fournir en quelques années la population ouvrière nécessaire à l'essor de ces véritables villes industrielles ; celles-ci se peuplent de ruraux en provenance de régions au sol moins fertile, souvent les hautes terres du Massif Central pour le Languedoc. Pendant longtemps, paysans et ouvriers constituent deux populations distinctes, souvent opposées par leurs cultures, langues, religions, opinions politiques, etc. qui ne se rencontrent guère qu'au marché. Il y a malgré tout des contacts - et même des mariages - et l'influence des agglomérations industrielles sur leur environnement est loin d'être négligeable. Cela se voit aussi bien dans l'architecture où l'emploi de la brique, de la tuile ou des voûtains en arc segmentaire en brique se diffuse autour des constructions industrielles, que dans la structure globale de la société; la nouvelle ville a besoin de services, publics ou privés (commerces, artisans...) qui font souvent appel à la population des villages voisins : il est nettement plus rentable d'être menuisier ou tailleur dans une ville nouvelle ouvrière que dans un village de versant, 500 mètres plus haut. Ces nouveaux centres provoquent ainsi un exode rural de proximité préjudiciable aux villages les plus proches.

37 On notera que ces implantations s'accompagnent obligatoirement d'une liaison ferroviaire ce qui renforce le rôle que joue l'industrialisation dans l'évolution régionale. On sait, par contre, que la mine et l'industrie lourde repoussent le textile qui supporte 
mal la pollution de l'air et de l'eau, conduisant à une spécialisation des vallées en fonction de la nature de leur sous-sol.

Si la plupart des installations minières ont été aujourd'hui détruites, deux groupes d'éléments paysagers viennent nous rappeler cette épopée.

Les terrils et crassiers où s'accumulaient stériles de la mine et scories des usines forment parfois de véritables montagnes, tout juste plus discrètes que dans le Nord car moins faciles à identifier dans le paysage. Ce sont souvent aussi des versants de débris, trop réguliers pour être naturels, des coulées de blocs où la végétation ne parvient pas à reprendre pied quand certains composants chimiques toxiques (soufre, métaux lourds) sont trop abondants.

Les forêts de pins! Le boisage des galeries et le soutènement des chantiers de taille consommaient énormément de bois. Dès 1835 environ, les compagnies exploitantes plantent sur les terres qu'elles ont acquises des arbres dont le bois est destiné à finir au fond de la mine. Après quelques essais, le choix s'est porté en Languedoc sur le pin maritime, bien adapté au climat, peu exigeant en matière de sol et dont la croissance rapide assure une bonne rentabilité des plantations. Jusque dans les années 1960, la mine utilise beaucoup de bois mais, depuis la fermeture des puits, la forêt abandonnée se développe au détriment de la chênaie et de la châtaigneraie voisines et couvre les versants d'un taillis serré propice à la propagation des incendies. Envahissante, la pinède minière continue à grignoter l'espace rural de la montagne cévenole.

\section{Les minéralisations dispersées}

41 Dans cette catégorie on peut réunir tous les gites dont le volume extrait reste modeste par rapport aux précédents.

Il peut s'agir de «micro-bassins " houillers, parfois limités à une ou deux couches exploitables sur les flancs d'une colline ou d'un vallon ou encore de filons ou d'amas métalliques : fer bien sûr, mais aussi plomb parfois argentifère, cuivre et plus rarement antimoine et même or, connus et souvent exploités artisanalement (parfois depuis l'Antiquité) tandis que le zinc, récemment découvert, donne lieu à une véritable « fièvre minière » dans les années 1870-1880.

43 Contrairement au groupe précédent, ces gisements apparaissent comme à peu près indépendants du relief et on peut aussi bien les rencontrer en fond de vallée ou à son voisinage que près des crêtes ou au milieu de versants escarpés : gisements de fer du Canigou (Pyrénées Orientales), mine de zinc des Malines à Saint-Laurent-le-Minier (Gard).

Quand le gisement est vraiment très restreint, le produit sommairement trié est directement expédié vers des usines centralisatrices plus ou moins proches. La commune ne bénéficie alors que de quelques emplois d'ouvriers pendant un petit nombre d'années. Ce n'est, en fait qu'une activité artisanale de plus qui ne modifie guère l'économie locale. S'il est plus conséquent, l'extraction elle-même est complétée par trois opérations complémentaires :

- concassage et tri grossier, le plus souvent au voisinage immédiat de la mine pour éviter le transport inutile des rebuts. Ce sont souvent les vestiges de ces bâtiments que l'on peut encore voir isolés en pleine montagne ; 
- le tri plus fin, souvent par voie humide (flottation, sluice box ${ }^{2}$ ) nécessite de l'eau et s'implante naturellement au pied de la mine, à l'endroit le plus proche bénéficiant de l'existence d'un ruisseau permanent ;

Figure 9

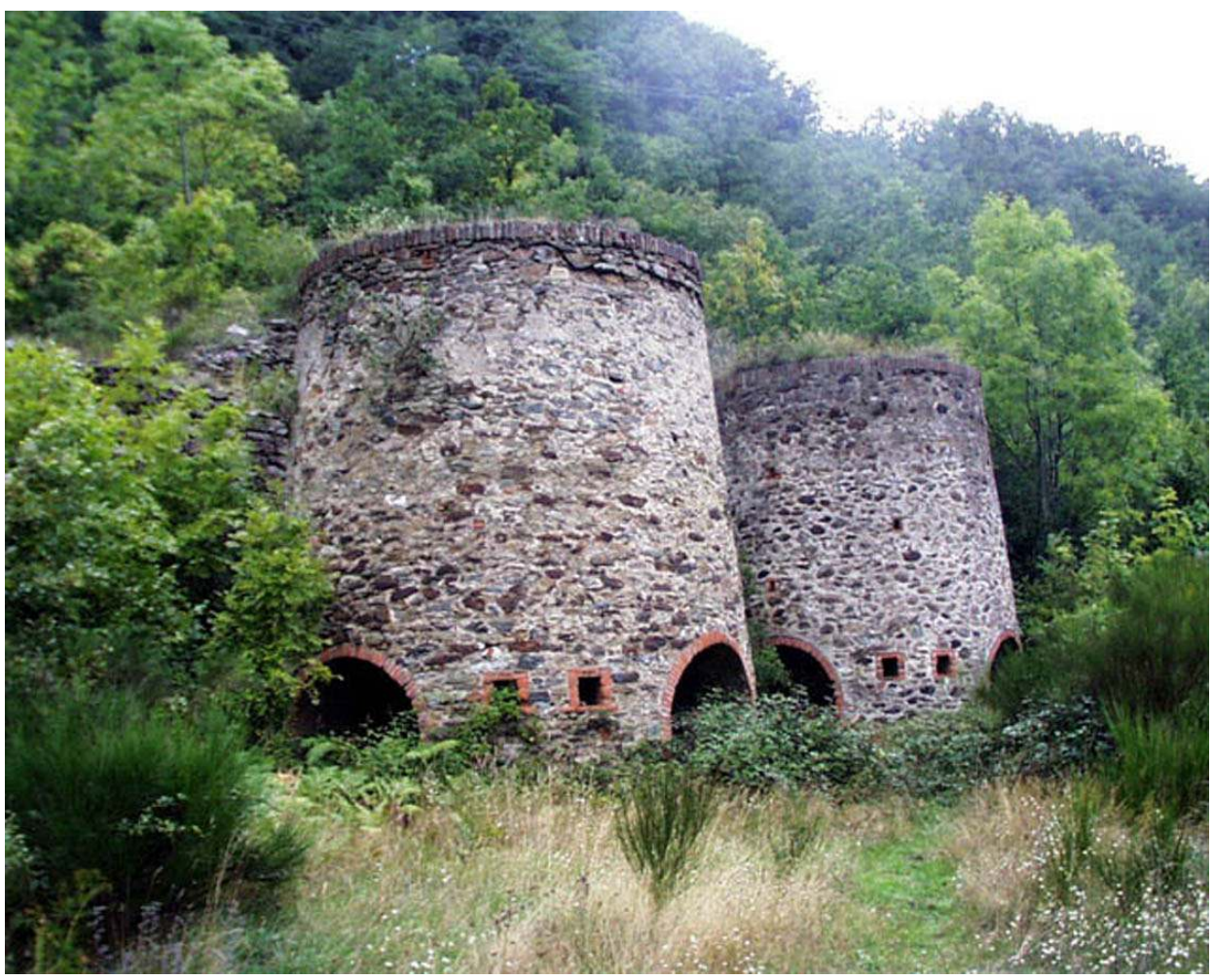

Fours de grillage du minerai de fer de Corsavy (Pyrénées Orientales), dans le massif du Canigou, Corsavy (Pyrénées Orientales). Wienin, Michel

(c) Wienin, Michel, 2002.

- le grillage, enfin, cuisson qui transforme les sulfures ou les carbonates hydratés en oxydes anhydres, faisant gagner jusqu'à $30 \%$ du poids (fig. $\mathbf{n}^{\circ} \mathbf{9 )}$ ). Tant que le transport du minerai s'effectue au sol (à dos d'homme, puis mulets, charrettes..), jusque vers 1880, les fours à grillage s'installent en contrebas immédiat des exploitations. Bois ou charbon nécessaires évitent le retour à vide des convois. Quand le transport par câble devient prépondérant, à la fin du XIXe siècle, la descente se fait par gravité, ce qui ne demande donc aucune énergie et les traitements vont s'implanter à l'arrivée du transporteur, en fond de vallée. Ainsi dans le massif du Canigou, les fours anciens se retrouvent dispersés dans la montagne tandis que ceux du XXe siècle sont à l'origine d'usines importantes dans les vallées (Arles-sur-Tech).

Enfin, les usines métallurgiques (fonderies, hauts fourneaux, etc.) ont d'abord été implantées dans les vallées proches des gisements et du bois longtemps indispensable à la transformation. Dans le Sud de la France, les forges «catalanes », mais aussi « rivoises » ou « biscayennes » ont assuré une production de fer exportée au loin. Pendant des siècles, leur «mail» (gros marteau hydraulique) et celui des martinets qui leur étaient souvent associés ont rythmé les vallées de leur martèlement puissant et continu. Le calme de ces endroits reculés si recherché de nos jours aurait paru celui de la mort à leurs habitants de 1800. Des installations plus performantes ont fait disparaître ces établissements à la limite de l'artisanat mais la tradition industrielle des vallées leur a survécu. 
Par la suite, le passage à la houille et au coke s'est souvent traduit par des implantations symétriques: un site sur le bassin houiller, un sur le minerai de fer tandis que l'électrométallurgie, consommatrice de puissances énergétiques considérables, s'est associée aux barrages, en particulier à ceux de haute chute.

47 Un dernier mot pour les produits de carrières, souvent volumineux et destinés à la pulvérisation, dont les usines de traitement sont systématiquement implantées au voisinage de rivières assez importantes pour emporter leur pollution, etc. Un bel exemple sur la haute vallée de l'Aude : les dolomies de Salvezines, exploitées au-dessus du village de ce nom sont traitées à une quinzaine de kilomètres de là, à Saint-Martin-Lys, au bord du fleuve.

\section{Lisibilité du patrimoine industriel montagnard}

La filature en bord de rivière, la cave coopérative d'affinage ou le four métallurgique abandonné sont facilement identifiables et plus souvent conservés au fond d'une forêt ou sur un versant inhospitalier qu'ils ne le seraient en périphérie urbaine, soumise à une pression foncière massive, mais ils ne sont que la partie évidente d'un patrimoine beaucoup plus large. Sans vouloir être exhaustif, on peut signaler deux domaines dans lesquels l'œil averti lira l'histoire industrielle des paysages : les aménagements linéaires et l'architecture d'habitat.

Les équipements linéaires tels que chemins, voies ferrées et plans inclinés ou canaux, avec leurs tracés parfois à flanc de falaise, leurs tunnels, leurs ponts et viaducs (fig. $\mathbf{n}^{\circ} \mathbf{1 0}$ ) , indispensables au fonctionnement des mines et des usines, ont souvent remplacé les anciens chemins et ont été conservés bien après l'arrêt de la production. Certains sont restés utilisés et d'autres, abandonnés, car désormais sans usage, ont parfois été repris à une date récente pour les besoins d'une nouvelle industrie : le tourisme (fig. $\left.\mathbf{n}^{\circ} \mathbf{1 1}\right)$. 
Figure 10

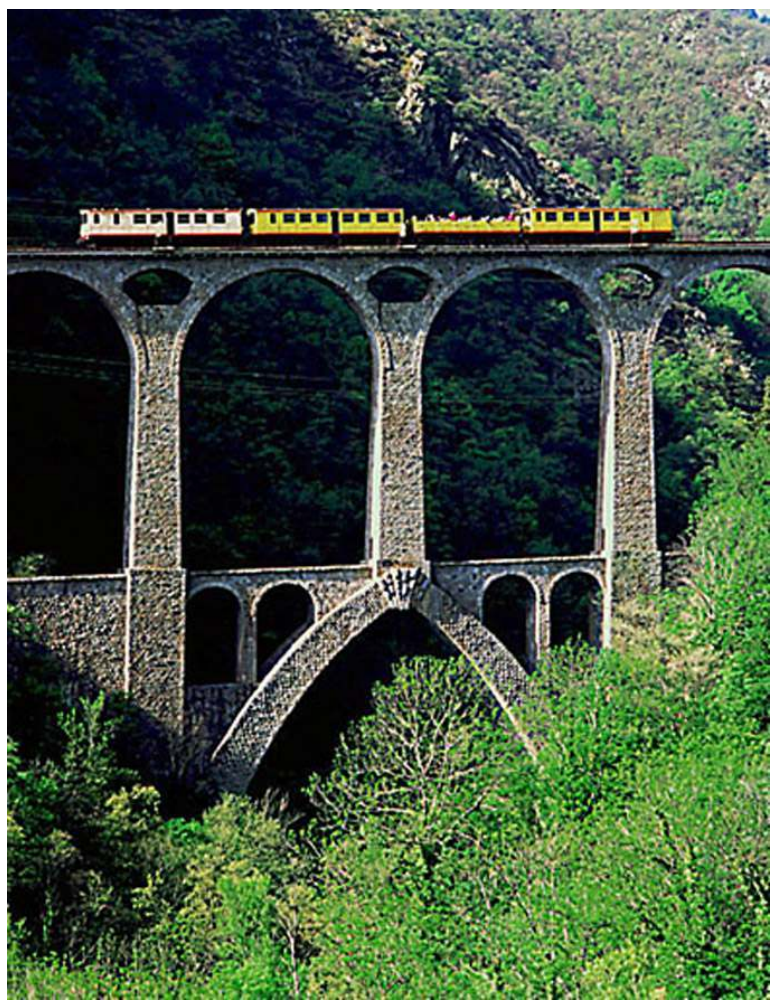

Viaduc Séjourné (protégé au titre des Monuments historiques) sur la ligne métrique de Cerdagne dite du «Train jaune ». Périn, Jean-Michel

(C) Inventaire général, ADAGP, 2000. 
Figure 11

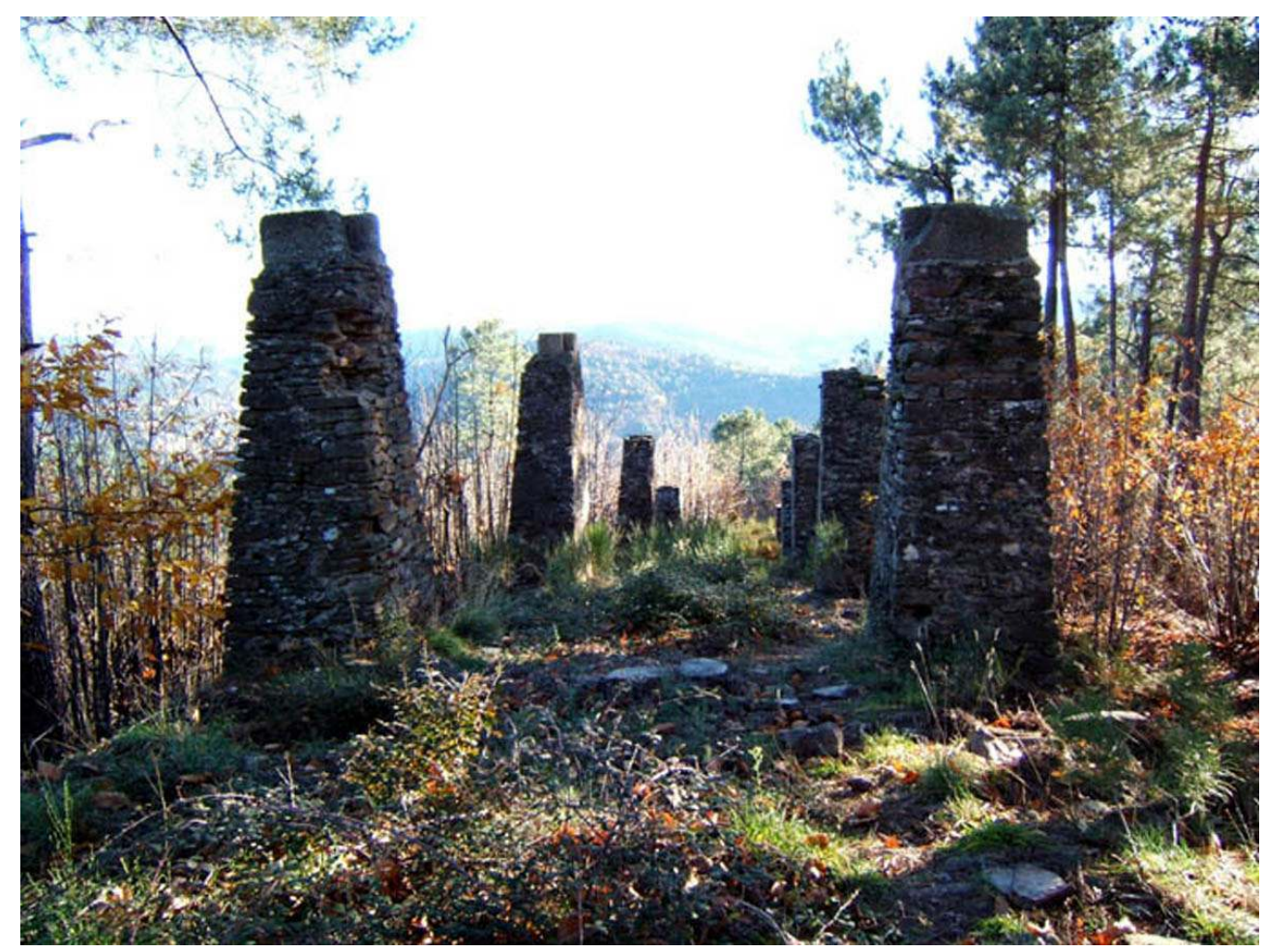

Piliers supportant les câbles d'un funiculaire de transport de la houille à Sainte-Cécile-d'Andorge (Gard) ; linéaire transformé en sentier de randonnée. Wienin, Michel

(c) Wienin, Michel, 2004

Quant à l'architecture urbaine, s'il est habituel aujourd'hui d'admirer la qualité architecturale des édifices agricoles, la majorité des touristes traversent les villages et les petites villes des fonds de vallées sans les voir, ou en en regardant seulement quelques bâtiments soigneusement sélectionnés : château, église, riche demeure, pont, lavoir... un moulin parfois. 


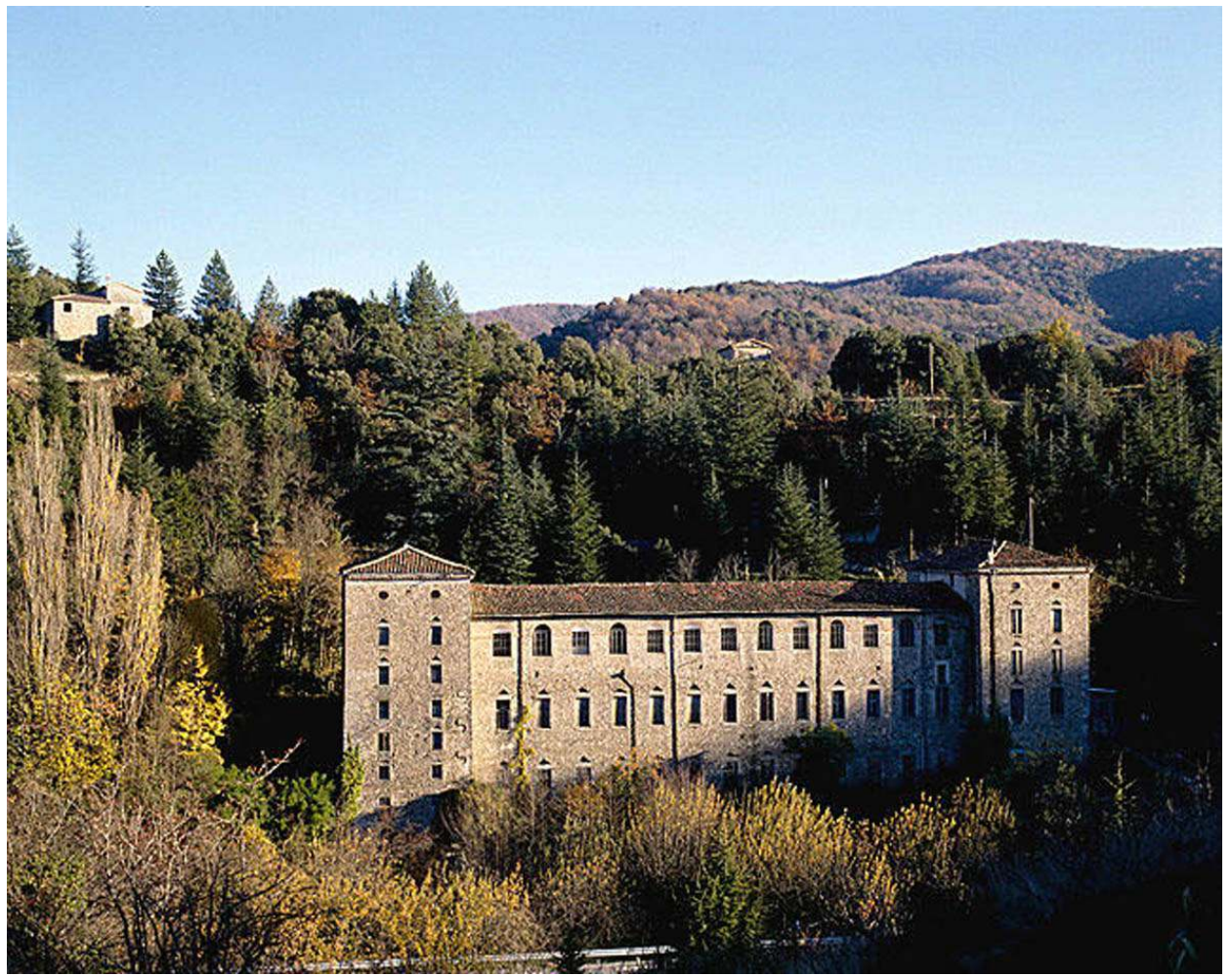

Filature de soie du Mazel à Notre-Dame-de-la-Rouvière (Gard), en partie visitable. Périn, Jean-Michel (c) Inventaire général, ADAGP, 1989.

On commence tout juste à signaler les quelques "rares » architectures industrielles conservées (fig. $\mathbf{n}^{\circ} \mathbf{1 2}$ ), pourtant souvent de qualité, et personne ne pense à montrer le fonds dominant, celui des maisons de rapport, des logements ouvriers du XIXe siècle. Maisons grises, hautes et percées de fenêtres nombreuses et régulières, elles s'apparentent d'un côté à la construction traditionnelle car réalisées par des maçons locaux, porteuses d'éléments décoratifs originaux (balcons sur voûtains, motifs ou encadrements en briques...) et de l'autre, correspondent à un autre mode de vie, non rural, (sans étable, grenier, séchoir ou silo, toutes ces choses qui font de la ferme un bâtiment utilitaire, adapté à une civilisation d'auto-production).

Prenons le temps de regarder ces maisons qui abritèrent une population laborieuse quand la montagne était industrielle, avant qu'elles ne soient frappées d'alignement pour élargir la route ou restaurées dans le goût des résidences secondaires.

\section{NOTES}

1. Le massif des Arbailles est un massif pyrénéen entre Basse-Navarre et Soule. 
2. Sluice box : " boîte à écluses ", c'est un canal incliné, généralement en bois, avec environ $10 \%$ de pente, où l'eau circule sans arrêt et qui peut mesurer plusieurs dizaines de mètres. Les particules de minerai, plus lourdes, sont piégées par des tasseaux ou d'autres structures de ralentissement disposés en travers tandis que les sables non métalliques, plus légers, sont entraînés.

\section{RÉSUMÉS}

\section{Michel Wienin}

Riches en énergie (rivières, bois, puis houille), en matières premières minérales et en produits d'élevage, les régions de montagne ont développé tôt une industrie qui profite de l'essor des transports au XIXe siècle. Loin des grands bassins miniers, les vallées se transforment en une succession de bourgs industriels, accédant beaucoup plus vite à la modernité que les plaines restées agricoles ou que les villages d'altitude, à l'écart du progrès. Délimité par le Massif Central et les Pyrénées, le Languedoc-Roussillon présente une plaine évoluant vers une quasimonoculture de la vigne et des vallées de montagne qui se tournent vers l'industrie textile et métallurgique.

France's mountain regions, which are rich in varied sources of energy (rivers, woods, then coal), in mineral resources and in products derived from livestock rearing, developed industries at an early date, benefiting from the development of new transport networks during the 19th century. Far from the major mining basins, the mountain valleys were transformed into a succession of industrial villages, reaching modernity more rapidly than the plains, still devoted to agriculture, or the villages high in the mountains and isolated from such progress. Bordered by the Massif Central and the Pyrenees, the region of Languedoc-Roussillon comprises a plain which evolved towards a mono-activity of wine production and the mountain valleys which witnessed the development of textile and metallurgical industries.

\section{INDEX}

Mots-clés : agroalimentaire, bois et dérivés, hydro-électricité, industrialisation, laine, Languedoc-Roussillon, métallurgie, mine, montagne, soie

Keywords : hydro-electricity, metallurgy, mining, mountains, silk, textile industry, woodworking, wool 